\title{
Derivative-expansion approach to the interaction between close surfaces
}

\author{
C. D. Fosco, ${ }^{1}$ F. C. Lombardo,,$^{2, *}$ and F. D. Mazzitelli ${ }^{1}$ \\ ${ }^{1}$ Centro Atómico Bariloche and Instituto Balseiro, Comisión Nacional de Energía Atómica, R8402AGP Bariloche, Argentina \\ ${ }^{2}$ Departamento de Física Juan José Giambiagi, FCEyN UBA and IFIBA CONICET-UBA, Facultad de Ciencias Exactas y Naturales, \\ Ciudad Universitaria, Pabellón I, 1428 Buenos Aires, Argentina
}

(Received 23 April 2014; published 30 June 2014)

\begin{abstract}
The derivative-expansion approach to the calculation of the interaction between two surfaces is a generalization of the proximity force approximation, a technique in widespread use in different areas of physics. The derivative expansion has so far been applied to seemingly unrelated problems in different areas; it is our principal aim here to present the approach in its full generality. To that end, we introduce a unified setting, which is independent of any particular application, provide a formal derivation of the derivative expansion in that general setting, and study some of its properties. With a view toward the possible application of the derivative expansion to other areas, like nuclear and colloidal physics, we also discuss the relation between the derivative expansion and some time-honored uncontrolled approximations used in these contexts. By putting them under similar terms as in the derivative expansion, we believe that the path is open to the calculation of next-to-leading-order corrections also for these contexts. We also review some results obtained within the derivative expansion, by applying it to different concrete examples and highlighting some important points.
\end{abstract}

DOI: 10.1103/PhysRevA.89.062120

PACS number(s): 12.20.Ds, 03.70.+k, 42.50.Ct

\section{INTRODUCTION}

The proximity force approximation (PFA) has been widely used in many areas of physics, as a tool to compute the total force between smooth surfaces at short distances, the scale of that smoothness being set by the distance between them. Although that total force may result from very different underlying microscopic mechanisms, the PFA is essentially the same in all of them, since its basis is geometrical.

The main idea behind this approximation was introduced by Derjaguin in 1934 [1], who studied the effect of contact deformations on the adhesion of particles. In that context, the so-called Derjaguin approximation (DA) has, as its main ingredient, the (assumed) knowledge of $E_{\|}(h)$, the interaction energy per unit area for two (infinite) plane parallel surfaces separated by a distance $h$. The DA tells us that then the interaction energy $U_{\mathrm{DA}}(d)$ between two curved surfaces is [1,2]

$$
U_{\mathrm{DA}}(d)=2 \pi R_{\mathrm{eff}} \int_{d}^{\infty} E_{\|}(h) d h,
$$

where $d$ is the distance between the surfaces, $R_{1}$ and $R_{2}$ are their respective curvature radii evaluated at the point of closest approach, and $R_{\text {eff }}=R_{1} R_{2} /\left(R_{1}+R_{2}\right)$. The same approximation can be alternatively written in terms of the force, $f_{\mathrm{DA}}$, as follows:

$$
f_{\mathrm{DA}}(d)=2 \pi R_{\mathrm{eff}} E_{\|}(d) .
$$

The usual derivation of this approximation relies upon the rather reasonable assumption that the interaction energy between the surfaces can be approximated by the PFA expression,

$$
U_{\mathrm{PFA}}=\int d S E_{\|},
$$

where the integration is performed over just one of the surfaces, or even over an intermediate mathematical surface

\footnotetext{
*lombardo@df.uba.ar
}

lying between the two physical surfaces. ${ }^{1}$ Assuming that the surfaces are gently curved, and approximating them by portions of the osculating spheres of radii $R_{1}$ and $R_{2}$ at the point of closest approach, one arrives at the DA.

The DA has been used to compute forces in many different physical situations: colloidal and macromolecular phenomena, nuclear physics, electrostatic forces, van der Waals interactions, Casimir forces, etc. The DA has been generally assumed to be an uncontrolled approximation, presumably working well for close and gently curved surfaces. Its major drawback was, perhaps, the absence of a procedure to assess the importance of the next-to-leading-order (NTLO) corrections since, by construction, the DA is not obtained as the leading term of any expansion. In spite of that drawback, surprisingly few works have been devoted to implementing a systematic improvement of the DA, which could give a more solid foundation for the PFA and a way to improve it.

Recently, we presented an approximation scheme, the so-called derivative expansion (DE), originally introduced within the context of the Casimir effect, for the calculation of the interaction energy between two surfaces, one of them flat and the other slightly curved [3,4]. This approximation has been shown to be a natural extension of the PFA, and it has proven to be useful in rather different situations, not just for Casimir effect calculations. The DE approach provides a systematic way of introducing the DA and the PFA and, in some circumstances, also of evaluating the NTLO corrections.

In this paper, our principal aim is to formulate and derive the DE in a quite general form, so that previous (and hopefully new) applications of it may be regarded as particular cases. To that end, we present a derivation of the PFA and its NTLO correction from first principles, for the particular case of a curved surface described by a function $x_{3}=\psi\left(x_{1}, x_{2}\right)$ in

\footnotetext{
${ }^{1}$ When applied to a compact object in front of a plane, the integration is usually restricted to the portion of the compact object's surface that faces the plane.
} 
front of a plane at $x_{3}=0$. We also interpret the result of this derivation in physical and geometrical terms.

The surfaces involved shall have quite different physical realizations, depending on the system considered. Indeed, in some examples they may correspond to two physical objects with very small widths, interacting as a result of the presence of an electric charge or dipole layers on them. In other cases, they may instead correspond to interfaces between different material media. Besides, the nature of the "microscopic" interaction that produces the interaction may also have quite different origins. For example, it may be electrostatic, mediated by a short-range force, or may even be the result of a more involved phenomenon, like the Casimir effect. As it stems from the different nature of the examples mentioned above, no assumption will be made about whether or not the interaction between the surfaces proceeds from a microscopic interaction that satisfies a superposition principle.

After presenting the general derivation, we touch on other efforts made in the literature towards generalizing and improving the DA, like the surface element integration (SEI) [5] and the surface integration approach (SIA) [6] introduced in the context of colloidal physics, as well as the different PFAs used in nuclear physics [7,8].

This paper is organized as follows: in Sec. II we provide a first-principles derivation of the DE and then a construction of it using physical arguments. We also consider some properties of the general formulas so obtained and comment on the form of the higher order terms. Then in Sec. III we discuss, from the point of view of the DE, some generalizations of the DA that have been proposed in the literature, mostly in the context of colloidal and nuclear physics. Based on a formal analogy with the Casimir interaction between almost-transparent media, we make contact with the SEI approach used in colloidal physics. In Sec. IV we briefly review results obtained during recent years using the DE, along with discussions of particular examples. Finally, Sec. V contains our conclusions.

\section{DERIVATION AND PROPERTIES OF THE DERIVATIVE EXPANSION}

\section{A. Derivation by resummation of a perturbative expansion}

Let us first set up the problem: regardless of the interaction considered, the geometry of the systemis assumed to be as follows: one of the surfaces, $L$, will be a plane, which (by a proper choice of coordinates) shall be described by the equation $x_{3}=0$. Denoting by $x_{1}, x_{2}$, and $x_{3}$ the set of three orthogonal Cartesian coordinates, the other surface, $R$, is assumed to be describable by using a single Monge patch. Namely, it can be parametrized in terms of just a single function $\psi\left(\mathbf{x}_{\|}\right)$, with $\mathbf{x}_{\|}=\left(x_{1}, x_{2}\right)$, such that $x_{3}=\psi\left(\mathbf{x}_{\|}\right)$.

To begin, we note that the interaction between the two surfaces shall be a functional $F[\psi]$ of a single function, $\psi\left(\mathbf{x}_{\|}\right)$. That functional may be an energy, a free energy, a force, etc., depending on the context and the kind of system being considered.

The PFA to $F$, which is denoted $F_{0}$ here, is obtained by adding, for each point $\mathbf{x}_{\|}$, the product of a local surface density $\mathcal{F}_{0}\left(\psi\left(\mathbf{x}_{\|}\right)\right)$, depending only on the value of $\psi$ at point $\mathbf{x}_{\|}$, times the surface element area; namely,

$$
F_{0}[\psi]=\int d^{2} \mathbf{x}_{\|} \mathcal{F}_{0}\left(\psi\left(\mathbf{x}_{\|}\right)\right)
$$

The local surface density is in turn determined by the knowledge of the exact form of $F$ for the case of two parallel surfaces,

$$
\mathcal{F}_{0}(a)=\lim _{\mathcal{S} \rightarrow \infty}\left[\frac{F[a]}{\mathcal{S}}\right],
$$

where $\mathcal{S}$ denotes the area of the $L$ plate.

Namely, to determine the density, one only needs to know the functional $F$ for constant functions $\psi \equiv a$ and then extract a surface factor due to translation invariance. This density is then evaluated at the local distance between plates, $\psi\left(\mathbf{x}_{\|}\right)$, multiplied by $d^{2} \mathbf{x}_{\|}$, and integrated. When the functional $F$ describes the interaction energy between the surfaces, the local density $\mathcal{F}_{0}$ is just the interaction energy per unit area $E_{\|}$, and $F_{0}$ becomes $U_{\text {PFA }}$ [see Eq. (3)].

We now show that one can derive the PFA and its corrections by considering

$$
\psi\left(\mathbf{x}_{\|}\right)=a+\eta\left(\mathbf{x}_{\|}\right)
$$

and performing a resummation of the perturbative expansion in powers of $\eta$. We start by expanding the functional $F[\psi]$ in powers of $\eta$,

$$
\begin{aligned}
F[\psi]= & \mathcal{S F}_{0}(a)+\sum_{n \geqslant 1} \int \frac{d^{2} k_{\|}^{(1)}}{(2 \pi)^{2}} \cdots \frac{d^{2} k_{\|}^{(n)}}{(2 \pi)^{2}} \delta\left(k_{\|}^{(1)}+\cdots\right. \\
& \left.+k_{\|}^{(n)}\right) h^{(n)}\left(k_{\|}^{(1)}, \ldots, k_{\|}^{(n)}\right) \widetilde{\eta}\left(k_{\|}^{(1)}\right) \cdots \widetilde{\eta}\left(k_{\|}^{(n)}\right),
\end{aligned}
$$

where the form factors $h^{(n)}$ can be computed by using standard perturbative techniques [9]. They may depend on $a$, although we do not write this dependence explicitly in order to simplify the notation.

Now we see that, for a smooth function $\eta$, the Fourier transform $\tilde{\eta}$ will be peaked at zero momentum; therefore, inside Eq. (7) we can approximate $h^{(n)}\left(k_{\|}^{(1)}, \ldots, k_{\|}^{(n)}\right) \simeq$ $h^{(n)}(0, \ldots, 0)$. As a consequence,

$$
F(\psi) \simeq \mathcal{S} \mathcal{F}_{0}(a)+\sum_{n \geqslant 1} h^{(n)}(0, \ldots, 0) \int d^{2} \mathbf{x}_{\|} \eta\left(\mathbf{x}_{\|}\right)^{n} .
$$

In principle, one could evaluate the form factors at zero momentum explicitly. However, there is a shortcut that allows one to obtain all of them at once: for a constant $\eta\left(\mathbf{x}_{\|}\right)=\eta_{0}$, the interaction energy is simply given by Eq. (8) with the replacement $\int d^{2} \mathbf{x}_{\|} \eta\left(\mathbf{x}_{\|}\right)^{n} \rightarrow \mathcal{S} \eta_{0}^{n}$. But for this particular case, $F$ is just the functional for parallel plates separated by a distance $a+\eta_{0}$, namely,

$$
\mathcal{F}_{0}\left(a+\eta_{0}\right)=\mathcal{F}_{0}(a)+\sum_{n \geqslant 1} h^{(n)}(0, \ldots, 0) \eta_{0}^{n} .
$$

Therefore, in this low-momentum approximation, the perturbative series can be summed up, the result being

$$
F_{0}[\psi] \simeq \int d^{2} \mathbf{x}_{\|} \mathcal{F}_{0}\left(a+\eta\left(\mathbf{x}_{\|}\right)\right)=\int d^{2} \mathbf{x}_{\|} \mathcal{F}_{0}(\psi),
$$

which is just the PFA. 
The straightforward calculation above has shown that, for the class of geometries considered in this paper, the PFA can be derived from first principles by performing a resummation of the perturbative calculation for the case of almost-flat surfaces. The PFA will be well defined as long as the form factors $h^{(n)}\left(k_{\|}^{(1)}, \ldots, k_{\|}^{(n)}\right)$ have a finite limit as $k_{\|}^{(i)} \rightarrow 0$.

This procedure also suggests that the PFA can be improved by considering the NTLO in the expansion of the form factors. We assume that the form factors can be expanded in powers of the momenta up to order 2 . This is a nontrivial assumption: depending on the details of the interaction considered, the low-momentum behavior of the form factors could include nonanalyticities. When that is not the case, we can perform the expansion

$$
\begin{aligned}
h^{(n)}\left(k_{\|}^{(1)}, \ldots, k_{\|}^{(n)}\right)= & h^{(n)}(0, \ldots, 0)+\sum_{i, \alpha} A_{i \alpha}^{(n)} k_{\| \alpha}^{(i)} \\
& +\sum_{i, j, \alpha, \beta} B_{i j \alpha \beta}^{(n)} k_{\| \alpha}^{(i)} k_{\| \beta}^{(j)} \ldots
\end{aligned}
$$

for some $a$-dependent coefficients $A_{i \alpha}^{(n)}$ and $B_{i j \alpha \beta}^{(n)}$. Here $i, j=$ $1, \ldots, n$ denote the different arguments of the form factor, and $\alpha, \beta=1,2$ their components. Symmetry considerations allow us to simplify the above expression. Indeed, rotational invariance implies that the form factors depend only on the scalar products $k_{\|}^{(i)} \cdot k_{\|}^{(j)}$. Moreover, they must be symmetric under the interchange of any two momenta. As a consequence,

$$
\begin{aligned}
h^{(n)}\left(k_{\|}^{(1)}, \ldots, k_{\|}^{(n)}\right)= & h^{(n)}(0, \ldots, 0)+B^{(n)} \sum_{i} k_{\|}^{(i) 2} \\
& +C^{(n)} \sum_{i \neq j} k_{\|}^{(i)} \cdot k_{\|}^{(j)}
\end{aligned}
$$

for some coefficients $B^{(n)}$ and $C^{(n)}$. Inserting Eq. (12) into Eq. (7), and performing integrations by parts, we find the following correction to the PFA:

$$
F_{2}[\psi]=\int d^{2} \mathbf{x}_{\|}\left[\sum_{n \geqslant 2} D^{(n)} \eta^{n-2}\right]|\nabla \eta|^{2},
$$

where the coefficients $D^{(n)}$ are linear combinations of $B^{(n)}$ and $C^{(n)}$. The subscript index 2 indicates the number of derivatives.

To complete the calculation, the next step is to calculate the sum in Eq. (13). This can be done by evaluating the correction $F_{2}$ for the particular case $\eta\left(\mathbf{x}_{\|}\right)=\eta_{0}+\epsilon\left(\mathbf{x}_{\|}\right)$, with $\epsilon \ll \eta_{0}$, and expanding up to second order in $\epsilon$. For this particular case,

$$
F_{2}\left[a+\eta_{0}+\epsilon\right]=\int d^{2} \mathbf{x}_{\|}\left[\sum_{n \geqslant 2} D^{(n)} \eta_{0}^{n-2}\right]|\nabla \epsilon|^{2} .
$$

Once more, the resummation can be performed, in this case by considering the usual perturbative evaluation of the interaction energy up to second order in $\epsilon$. This calculation will depend on the particular interaction considered, and from the result, one can obtain the series above, which we denote $Z$. More explicitly,

$$
Z\left(a+\eta_{0}\right) \equiv \sum_{n \geqslant 2} D^{(n)} \eta_{0}^{n-2} .
$$

Making the replacement $\eta_{0} \rightarrow \eta$ in the above equation, we arrive at

$$
F_{2}[\psi]=\int d^{2} \mathbf{x}_{\|} Z(\psi)|\nabla \psi|^{2},
$$

which is the NTLO correction to the PFA. This concludes the derivation of the PFA and its first correction, which reads

$$
F_{\mathrm{DE}}[\psi]=d^{2} \mathbf{x}_{\|}\left[V(\phi)+Z(\psi)|\nabla \psi|^{2}\right],
$$

where $V(\psi)=\mathcal{F}_{0}(\psi)$ is determined from the known value of the parallel surfaces geometry, while $Z(\psi)$ can be computed perturbatively. Note that $Z$ can be evaluated, in practice, by just setting $\eta_{0}=0$ in Eq. (15).

Higher orders may be derived by a natural extension of the procedure. It also becomes apparent that for the expansion to be well defined, the analytic structure of the form factors appearing in the perturbative expansion around flat surfaces is relevant. In particular, the existence of zero-momentum singularities can certainly render the DE nonapplicable; on the other hand, this should be expected on physical grounds since these singularities imply that the functional cannot be approximated, in coordinate space, by the single integral of a local density. Physically, it is a signal that the interaction becomes essentially nonlocal (see Sec. IV C 2). Indeed, if written in coordinate space, they would require more than one integral over the spatial coordinates.

\section{B. Construction of the second-order derivative expansion}

We now present a physical construction of the DE, based on a procedure whereby one attempts to improve the PFA. The expression for the PFA does not involve derivatives of $\psi$, since one is using parallel planes to obtain the density, and the corresponding functional is characterized by a single number, their distance. The DE may then be introduced, as a rather natural way to improve the PFA, simply by constructing an improved surface density $\mathcal{F}$. The improvement can be implemented by using the density that results from using, at each point of the surface, a second-order approximation to it. Namely, the curved surface shall be approximated (locally) by a surface that makes a second-order contact with it, i.e., that has the same first and second derivatives as $\psi$.

In other words, rather than evaluating $F$ for a constant $\psi=a$, we consider evaluating it at

$$
\psi=\psi\left(\mathbf{y}_{\|}\right)=a+\eta\left(\mathbf{y}_{\|}\right),
$$

where $\eta$ is a quadratic function of its argument:

$$
\eta\left(\mathbf{y}_{\|}\right)=\sum_{i=1}^{2} b_{i} y_{i}+\frac{1}{2} \sum_{i, j=1}^{2} c_{i j} y_{i} y_{j} .
$$

We use $\mathbf{y}_{\|}$for the coordinates on which $\eta$ may depend, since later on we use $\mathbf{x}_{\|}$to denote each point on which the expansion is performed (for example, $a$ will depend on $\mathbf{x}_{\|}$).

Since we want to construct a second-order expansion in derivatives, the expression for $F$ will be expanded up to the second order in $b_{i}$ and first order in $c_{i j}$. That expansion shall have the form

$$
F=F^{(0)}+F^{(1)}+F^{(2)}+\cdots,
$$


where the index denotes the order in derivatives. One then has $F^{(0)}=F[a]$ (yielding the PFA contribution), while the term of order 1 in derivatives is

$$
F^{(1)}=\int d^{2} \mathbf{y}_{\|} \Gamma_{a}^{(1)}\left(\mathbf{y}_{\|}\right) \sum_{i=1}^{2} b_{i} y_{i},
$$

with

$$
\Gamma_{a}^{(1)}\left(\mathbf{y}_{\|}\right)=\left[\frac{\delta F[a+\eta]}{\delta \eta\left(\mathbf{y}_{\|}\right)}\right]_{\eta=0} .
$$

The second-order term receives two contributions:

$$
\begin{aligned}
F^{(2)}= & \int d^{2} \mathbf{y}_{\|} \Gamma_{a}^{(1)}\left(\mathbf{y}_{\|}\right)\left(\frac{1}{2} \sum_{i, j=1}^{2} c_{i j} y_{i} y_{j}\right)+\frac{1}{2} \int d^{2} \mathbf{y}_{\|} \\
& \times \int d^{2} \mathbf{y}_{\|}^{\prime}\left(\sum_{i=1}^{2} b_{i} y_{i}\right) \Gamma_{a}^{(2)}\left(\mathbf{y}_{\|}, \mathbf{y}_{\|}^{\prime}\right)\left(\sum_{j=1}^{2} b_{j} y_{j}^{\prime}\right),
\end{aligned}
$$

one involving the one-point kernel appearing in $F^{(1)}$ and the other depending on the two-point function,

$$
\Gamma_{a}^{(2)}\left(\mathbf{y}_{\|}, \mathbf{y}_{\|}^{\prime}\right)=\left[\frac{\delta^{2} F(a+\eta)}{\delta \eta\left(\mathbf{y}_{\|}\right) \delta \eta\left(\mathbf{y}_{\|}^{\prime}\right)}\right]_{\eta=0} .
$$

Let is consider now the term of first order in derivatives, $F^{(1)}$. We first see that $\Gamma_{a}^{(1)}$ cannot depend on $\mathbf{y}_{\|}$(since the functional derivative is evaluated at a constant function), and it is therefore a constant regarding that variable. Indeed, note that it can even be written explicitly in terms of the density $\mathcal{F}_{0}(a)$ :

$$
\Gamma_{a}^{(1)}=\frac{1}{\mathcal{S}} \frac{\partial F[a]}{\partial a}=\frac{\partial \mathcal{F}_{0}(a)}{\partial a} .
$$

Then we see that

$$
F^{(1)}=F_{a}^{(1)} \int d^{2} \mathbf{y}_{\|} b_{i} y_{i}=0,
$$

thus the first-order term vanishes.

Let us now consider the two contributions to the secondorder term, $F^{(2)}$, namely, $F^{(2)}=F^{(2,1)}+F^{(2,2)}$, with

$$
F^{(2,1)}=\int d^{2} \mathbf{y}_{\|} \Gamma_{a}^{(1)}\left(\mathbf{y}_{\|}\right)\left(\frac{1}{2} \sum_{i, j=1}^{2} c_{i j} y_{i} y_{j}\right)
$$

and

$$
\begin{aligned}
F^{(2,2)}= & \frac{1}{2} \int d^{2} \mathbf{y}_{\|} \int d^{2} \mathbf{y}_{\|}^{\prime}\left(\sum_{i=1}^{2} b_{i} y_{i}\right) \\
& \times \Gamma_{a}^{(2)}\left(\mathbf{y}_{\|}, \mathbf{y}_{\|}^{\prime}\right)\left(\sum_{j=1}^{2} b_{j} y_{j}^{\prime}\right) .
\end{aligned}
$$

Regarding $F^{(1)}$, using (25), one sees that

$$
F^{(2,1)}=\mathcal{S} \frac{\partial \mathcal{F}_{0}(a)}{\partial a}\langle\eta\rangle,
$$

where we have introduced the spatial average of $\eta$,

$$
\langle\eta\rangle \equiv \frac{1}{\mathcal{S}} \int d^{2} \mathbf{y}_{\|} \eta\left(\mathbf{y}_{\|}\right) .
$$

Regarding $F^{(2,1)}$, since $\Gamma_{a}^{(2)}$ can only depend on the difference between its arguments,

$$
F^{(2,2)}=\frac{1}{2} \sum_{i, j=1}^{2} b_{i} b_{j} \int d^{2} \mathbf{y}_{\|} \int d^{2} \mathbf{y}_{\|}^{\prime} y_{i} \Gamma_{a}^{(2)}\left(\mathbf{y}_{\|}-\mathbf{y}_{\|}^{\prime}\right) y_{j}^{\prime},
$$

and in terms of $\widetilde{\Gamma}_{a}^{(2)}\left(\mathbf{k}_{\|}\right)$, the Fourier transform of $\Gamma_{a}^{(2)}$,

$$
\begin{aligned}
& \int d^{2} \mathbf{y}_{\|} \int d^{2} \mathbf{y}_{\|}^{\prime} y_{i} \Gamma_{a}^{(2)}\left(\mathbf{y}_{\|}-\mathbf{y}_{\|}^{\prime}\right) y_{j}^{\prime} \\
& =\mathcal{S} \lim _{\mathbf{k}_{\|} \rightarrow 0}\left[\frac{\partial^{2} \widetilde{\Gamma}_{a}^{(2)}\left(\mathbf{k}_{\|}\right)}{\partial k_{i} \partial k_{j}}\right] \\
& =\mathcal{S} \frac{1}{2} \delta_{i j} \lim _{\mathbf{k}_{\|} \rightarrow 0}\left[\sum_{i=1}^{2} \frac{\partial^{2} \widetilde{\Gamma}_{a}^{(2)}\left(\mathbf{k}_{\|}\right)}{\partial k_{i}^{2}}\right],
\end{aligned}
$$

where rotational invariance on the $x_{3}=a$ spatial planes has been used. Thus,

$$
F^{(2,2)}=\mathcal{S} Z(a) \sum_{i=1}^{2} b_{i}^{2}
$$

with

$$
Z(a)=\frac{1}{4} \lim _{\mathbf{k}_{\|} \rightarrow 0}\left[\sum_{j=1}^{2} \frac{\partial^{2}}{\partial k_{i}^{2}} \widetilde{\Gamma}_{a}^{(2)}\left(\mathbf{k}_{\|}\right)\right]
$$

Putting together the results for $F^{(0)}, F^{(1)}$, and $F^{(2)}$ we see that, in all of them, an $\mathcal{S}$ factors out. Therefore, up to second order in derivatives,

$$
F[a+\eta]=\mathcal{S}\left[\mathcal{F}_{0}(a)+\frac{\partial \mathcal{F}_{0}(a)}{\partial a}\langle\eta\rangle+Z(a) \sum_{i=1}^{2} b_{i}^{2}\right]
$$

or (since $\langle\eta\rangle$ involves two derivatives), to the same order,

$$
F[a+\eta]=\mathcal{S}\left[\mathcal{F}_{0}(a+\langle\eta\rangle)+Z(a) \sum_{i=1}^{2} b_{i}^{2}\right] .
$$

It then transpires that it is convenient to perform the splitting between $a$ and $\eta$ in such a way that $\langle\eta\rangle=0$, since then the $c_{i j}$ term may be ignored (to second order). In what follows, we assume that this is the case.

Thus, the second-order correction to the density, in the DE, is denoted $\mathcal{F}_{2}$ and shall be

$$
\mathcal{F}_{2}\left(a, b_{i}, c_{i j}\right)=Z(a) \sum_{i=1}^{2} b_{i}^{2},
$$

and the resulting contribution to $F$ results by integrating this density, evaluated at the proper ( $\mathbf{x}_{\|}$-dependent) values of $a$ and $b_{i}$ :

$$
F_{2}=\int d^{2} \mathbf{x}_{\|} Z(\psi)(\nabla \psi)^{2}
$$

The total $F$ in this second-order approximation is thus obtained by multiplying the two contributions to the density by the area element and integrating. This results in $F_{\mathrm{DE}}$, the functional given in Eq. (17), where, we recall, the two functions 
that completely determine the approximation are obtained from the functional $F$, as follows:

$$
V(\psi) \equiv \mathcal{F}_{0}\left(\psi\left(\mathbf{x}_{\|}\right)\right)=\left[\mathcal{F}_{0}(a)\right]_{a=\psi\left(\mathbf{x}_{\|}\right)}
$$

and

$$
Z(\psi)=\frac{1}{4} \lim _{\mathbf{k}_{\|} \rightarrow 0}\left[\sum_{j=1}^{2} \frac{\partial^{2}}{\partial k_{i}^{2}} \widetilde{F}_{a}^{(2)}\left(\mathbf{k}_{\|}\right)\right]_{a=\psi\left(\mathbf{x}_{\|}\right)} .
$$

Note that, from the formulas that determine the functions $V$ and $Z$, we see that a possible practical way to obtain them is by evaluating $F(\psi)$ for $\psi=a+\eta$, with an $\eta$ whose spatial average is 0 , and expanding $F$ up to the second order in $\eta$ and its derivatives. An important implicit assumption in the construction above is the existence of the zero-momentum limit in Eq. (32). This is of course the same assumption we made in the previous subsection about the analytic structure of the form factors, which in turn depend on the problem being considered. We remark once more on the fact that the nonexistence of that limit means that the functional $F$ shall receive nonlocal contributions beyond the PFA. In other words, it will not be just the integral of a density.

\section{Some general properties of the derivative expansion}

We present here some consequences that follow from the form of the general expression for the DE. Let us first point out an important property of both the PFA and its NTLO correction in the DE: they stem from the expansion in derivatives of a functional, thus they involve integrals of local functions of $\psi$ and its derivatives. Thus, in order to assess the relative magnitude of those terms, it is not sufficient, in general, to look at the behavior of the integrands at some particular points. Rather, they measure global features of the distance function $\psi$ and its derivatives. Thus, a function may have a small curvature radius at some region but, nevertheless, give rise to a small contribution. Besides, note that these two terms have different properties regarding spatial scaling. Indeed, let us consider the two terms $F_{0}[\psi]$ and $F_{2}[\psi]$ for a given function $\psi$, assuming that the corresponding integrals are finite. Then introduce the scaled function,

$$
\psi_{\lambda}\left(\mathbf{x}_{\|}\right) \equiv \psi\left(\lambda \mathbf{x}_{\|}\right)
$$

for a scale parameter $0<\lambda<\infty$. We see, by evaluating these terms for the scaled function, that

$$
F_{0}\left[\psi_{\lambda}\right]=\lambda^{-2} F_{0}[\psi], \quad F_{2}\left[\psi_{\lambda}\right]=F_{2}[\psi],
$$

as follows by performing the change of variables $\mathbf{x}_{\|} \rightarrow \lambda^{-1} \mathbf{x}_{\|}$ in both integrals. Thus, when the function $\psi$ has a bump or depression of characteristic size $r_{0}$, the second-order term will be independent of $r_{0}$, producing the same contribution, even when the size goes to 0 (and the corresponding curvature diverges).

This can be illustrated by an example which appears in Casimir physics. Assuming the boundary conditions are perfect, there is no dimensionful object in the system except $\psi$ itself. Thus, in natural units, when calculating the energy, both $V$ and $Z$ have to be proportional to $1 / \psi^{3}$. Here the role of the functional $F$ is played by the static energy, $U[\psi]$. The zeroth- and second-order terms in the DE for the energy then have the form

$$
\begin{aligned}
& U_{0}[\psi]=a_{0} \int d^{2} \mathbf{x}_{\|} \frac{1}{\psi^{3}}, \\
& U_{2}[\psi]=a_{2} \int d^{2} \mathbf{x}_{\|} \frac{|\nabla \psi|^{2}}{\psi^{3}},
\end{aligned}
$$

where $a_{0}$ and $a_{2}$ are constants.

For the revolution paraboloid $\psi=a\left(1+\frac{\mathbf{x}_{\|}^{2}}{\sigma^{2}}\right)$, we may evaluate both terms exactly, which yields

$$
\begin{aligned}
& U_{0}[\psi]=\frac{\pi}{2} a_{0} \frac{\sigma^{2}}{a^{3}}, \\
& U_{2}[\psi]=2 \pi a_{2} \frac{1}{a} .
\end{aligned}
$$

Since $\sigma$ is proportional to the radius of curvature, the ratio $\frac{\sigma^{2}}{a^{2}} \gg 1$, and therefore the second-order term is much smaller than the zeroth-order one.

This analysis can be extended to the higher order terms. Indeed, a term with $2 n(n \in \mathbb{N})$ derivatives has in the integrand a $1 / \psi^{3}$ factor times a polynomial with $2 n$ derivatives and $2 n$ powers of $\psi$ in the numerator. Thus, under scaling,

$$
U_{2 n}\left[\psi_{\lambda}\right]=\lambda^{2 n-2} U_{2 n}[\psi] .
$$

Thus, for the quadratic $\psi$ that we are considering,

$$
U_{2 n} \propto \frac{1}{a}\left(\frac{a}{\sigma}\right)^{2 n-2} .
$$

Of course, we have assumed the expansion to be well defined to those orders; that is something which, as we shall see, depends on the properties of the system.

\section{Fourth-order term}

Let us, for the sake of illustration, briefly comment here on the construction of the fourthorder term (there is no third-order one), in a scheme similar to the one considered in Sec. IIB. To derive the improved $F$ density, one now has to consider

$$
\psi=\psi\left(\mathbf{y}_{\|}\right)=a+\eta\left(\mathbf{y}_{\|}\right),
$$

where

$$
\begin{aligned}
\eta\left(\mathbf{y}_{\|}\right)= & \sum_{i=1}^{2} b_{i} y_{i}+\frac{1}{2} \sum_{i, j=1}^{2} c_{i j} y_{i} y_{j}+\frac{1}{3 !} \sum_{i, j, k=1}^{2} d_{i j k} y_{i} y_{j} y_{k} \\
& +\frac{1}{4 !} \sum_{i, j, k, l=1}^{2} e_{i j k l} y_{i} y_{j} y_{k} y_{l} .
\end{aligned}
$$

To find the fourth-order term in derivatives, one should collect in $F$ terms which come from its first-order contribution in $e$, second order in $c$, fourth order in $b$, and also first order in $b$ and $d$ or second order in $b$ and first in $c$.

Thus $F^{(4)}$ receives five different contributions, $F^{(4, j)}(j=$ $1, \ldots, 5)$. All these contributions may be evaluated in Fourier space, where they can be expressed in terms of derivatives at zero momentum of the corresponding functional derivative; besides, these functional derivatives, being evaluated at $\eta=0$, are translation invariant. 
It is possible to check that the $F^{(4,1)}$ term produces a constant, and it can be ignored at this order by a redefinition of $a$. The remaining terms have the structure

$$
\begin{gathered}
F^{(4,2)}=\frac{1}{8} \mathcal{S} \sum_{i, j, k, l}^{2} \gamma_{i j k l}^{(4,2)} c_{i j} c_{k l}, \\
F^{(4,3)}=\frac{1}{24} \mathcal{S} \sum_{i, j, k, l}^{2} \gamma_{i j k l}^{(4,3)} b_{i} b_{j} b_{k} b_{l}, \\
F^{(4,4)}=\frac{1}{6} \mathcal{S} \sum_{i, j, k, l}^{2} \gamma_{i j k l}^{(4,4)} b_{i} b_{j} c_{k l}, \\
F^{(4,5)}=\frac{1}{4} \mathcal{S} \sum_{i, j, k, l}^{2} \gamma_{i j k l}^{(4,5)} b_{i} d_{j k l},
\end{gathered}
$$

where the $\gamma$ coefficients are constant tensors, which, moreover, may be further simplified by using rotational invariance. All the terms carry a factor of $\mathcal{S}$, which appears because of momentum conservation.

We conclude by analyzing the tensor corresponding to one of these contributions: $\gamma_{i j k l}^{(4,2)}$. It can be obtained as

$$
\gamma_{i j k l}^{(4,2)}=\left[\frac{\partial^{4} \widetilde{\Gamma}^{(2)}(p)}{\partial p_{i} \partial p_{j} \partial p_{k} \partial p_{l}}\right]_{p \rightarrow 0},
$$

and rotational invariance means that it has the form

$$
\gamma_{i j k l}^{(4,2)}=C\left(\delta_{i j} \delta_{k l}+\delta_{i k} \delta_{j l}+\delta_{i l} \delta_{i j} \delta_{j k}\right),
$$

where $C$ is an $a$-dependent constant, determined by the term of order 4 in an expansion of the kernel at low momenta:

$$
C=\frac{1}{8}\left[\frac{\partial^{4} \widetilde{\Gamma}^{(2)}(p)}{\partial p_{i} \partial p_{i} \partial p_{j} \partial p_{j}}\right]_{p \rightarrow 0} .
$$

When used to construct the DE to fourth order, this term shall produce

$$
F^{(4,2)}=\frac{1}{8} \int d^{2} \mathbf{x}_{\|} C(\psi)\left[|\nabla \psi|^{4}+2\left(\partial_{i} \partial_{j} \psi\right)^{2}\right] .
$$

A similar approach allows one to derive all the other contributions.

\section{GENERALIZATIONS OF THE DERJAGUIN APPROXIMATION IN NUCLEAR AND COLLOIDAL PHYSICS}

In this section we discuss some generalizations of the DA proposed in the context of nuclear and colloidal physics, from the point of view of the DE.

\section{A. Generalized PFA in nuclear physics}

The application of the DA in nuclear physics started with a celebrated paper by Blocki et al. [7]. In that paper, the authors rediscovered the DA in a rather different context and applied it to compute the interaction between nuclei. The starting point of Ref. [7] is a Derjaguin-like formula for the interaction energy between surfaces. That formula incorporates, as an essential ingredient, what the authors called 'universal function', the interaction energy between planar surfaces, which the authors calculated using a Thomas-Fermi approximation.

To proceed, let us describe the kind of system being considered and, at the same time, introduce some notation: let us consider two surfaces $S_{L}$ and $S_{R}$, plus an intermediate mathematical surface $S$ used to parametrize the physical ones. For smooth and slightly curved surfaces, we expect the interaction energy to be well described by the PFA, as in Eq. (3). One can now rewrite the surface integral above by introducing the set of level curves for $h$ in $S$ : they are closed curves that correspond to a fixed distance $h$ between $S_{L}$ and $S_{R}$. Denoting by $J(h) d h$ the area between two curves on $S$ corresponding to distances $h$ and $h+d h$, the PFA expression for the interaction energy $U$ can be written as a one-dimensional integral:

$$
U_{\mathrm{PFA}}=\int d h J(h) E_{\|}(h) .
$$

We then assume the surfaces to be gently curved, so that just one patch is sufficient to describe them, and besides, we use Cartesian coordinates $\left(x_{1}, x_{2}\right) \equiv \mathbf{x}_{\|}$on $S$. The distance between $S_{L}$ and $S_{R}$ then becomes a function $h=h\left(\mathbf{x}_{\|}\right)$, and $J$ is constant.

Performing a second-order Taylor expansion of $h$ around the point of closed approach, which corresponds to a distance $d$,

$$
h\left(x_{1}, x_{2}\right) \simeq d+\frac{1}{2} \frac{x_{1}^{2}}{R_{1}}+\frac{1}{2} \frac{x_{2}^{2}}{R_{2}},
$$

where $R_{1}$ and $R_{2}$ are the radii of curvature of the surface defined by $x_{3}=h\left(\mathbf{x}_{\|}\right)$, one obtains the DA of Eq. (1).

In a subsequent paper [8], a generalization of the PFA was introduced. The starting point was again Eq. (57), but now the surfaces could have large curvatures, as long as they remained almost parallel locally. The main difference introduced by the weaker assumptions about the surfaces is that now the Jacobian $J$ may become a nontrivial function of $h$, rather than being just a constant. Using the linear expansion

$$
J(h) \approx J_{0}+J_{1} h
$$

it can be shown that the force $f$ between surfaces becomes

$$
f_{\mathrm{PFA}}(d)=J_{0} E_{\|}(d)-J_{1}(d) \int_{d}^{\infty} d h E_{\|}(h),
$$

where the second term is a correction to the usual DA. Note, however, that from a conceptual point of view, this is not a generalization of the DA, since the starting point is the same as before: $U_{\mathrm{PFA}}$. What the previous formula does is to provide an explicit formula for the surface integral appearing in the PFA, which now involves a new geometrical object, the Jacobian $J$.

In other words, Eq. (60) is still determined by the energy density for parallel plates, not including corrections to that object, like the ones appearing in the DE. That kind of correction depends on the geometry and on the nature of the interaction.

Note that in nuclear physics there is an additional complication to deal with: even for two infinite half-spaces separated by a gap, the interaction energy $E_{\|}$is not exactly known. Different approximations have been used to compute that, and they give rise to different PFAs. For a recent review see Ref. [10]. 


\section{B. SEI and SIA in colloidal physics}

The SEI [5] and SIA [6] methods have been introduced within the context of colloidal physics and constitute another generalization of the DA. While based on different physical assumptions, the final result in both cases is the same. It may be introduced as follows: consider a compact object in front of a plane $x_{3}=0, x_{3}$ denoting the normal coordinate to the plane that points towards the compact object.

The SEI approximation to the interaction energy is then given by

$$
U_{\mathrm{SEI}}=-\int_{\text {plane }} d x_{1} d x_{2} \frac{\hat{n} \cdot \hat{e}_{3}}{\left|\hat{n} \cdot \hat{e}_{3}\right|} E_{\|},
$$

where $\hat{n}$ is the unit outward normal to a surface element in the compact object. When the compact object can be thought of as being delimited by two surfaces, one facing the plane and the other away from it, the SEI approximation consists in computing the difference between the usual PFAs for each surface.

It may appear surprising, at first glance, that the two surfaces contribute with different signs to the interaction energy. However, as we will see, this is related to the fact that the SEI becomes exact for almost-transparent bodies, where the interaction comes from volumetric pairwise contributions.

In the colloidal physics literature, the SEI method is justified by assuming that there is a pressure on the compact object, which should be integrated over the closed surface surrounding it in order to find the total force [5]. Alternatively, it has been shown that Eq. (61) becomes exact when the interaction between bodies can be obtained as the result of pair potentials of their constituents [6].

In order to understand, and reinterpret, this formula within the context of Casimir physics and, at the same time, to provide a systematic way of evaluating the NTLO, we use a rather simple example. Let us consider a quantum scalar field $\varphi$, in the presence of two media: one of them, denoted by $L$, corresponding to the $x_{3} \leqslant 0$ half-space, while the other, $R$, is defined in terms of two functions,

$$
R=\left\{\left(x_{1}, x_{2}, x_{3}\right): \psi_{1}\left(x_{1}, x_{2}\right) \leqslant x_{3} \leqslant \psi_{2}\left(x_{1}, x_{2}\right)\right\},
$$

as shown in Fig. 1.

Besides, we assume that the field propagation inside each medium can be represented by the presence of a nonvanishing interaction term. Assuming also that outside the $L$ and $R$ regions there is vacuum, the Euclidean action adopts the form

$$
\mathcal{S}[\varphi]=\mathcal{S}_{0}[\varphi]+\mathcal{S}_{I}[\varphi],
$$

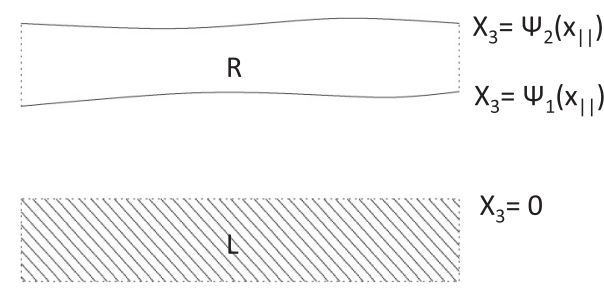

FIG. 1. Representation of the two media. One of them, denoted $L$, corresponds to the $x_{3} \leqslant 0$ half-space, while the other, $R$, is defined in terms of two functions: $R=\left\{\left(x_{1}, x_{2}, x_{3}\right): \psi_{1}\left(x_{1}, x_{2}\right) \leqslant\right.$ $\left.x_{3} \leqslant \psi_{2}\left(x_{1}, x_{2}\right)\right\}$. where

$$
\mathcal{S}_{0}[\varphi]=\frac{1}{2} \int d^{4} x(\partial \varphi)^{2}
$$

is the free action for the fluctuating vacuum field, while $\mathcal{S}_{I}$ contains two terms, corresponding to the $L$ and $R$ regions, respectively,

$$
\mathcal{S}_{I}[\varphi]=\mathcal{S}_{L}[\varphi]+\mathcal{S}_{R}[\varphi],
$$

with

$$
\mathcal{S}_{L}[\varphi]=\frac{\lambda_{L}}{2} \int d^{3} x_{\|} \int_{-\infty}^{0} d x_{3} \mathcal{L}_{I}(\varphi)
$$

and

$$
\mathcal{S}_{R}[\varphi]=\frac{\lambda_{R}}{2} \int d^{3} x_{\|} \int_{\psi_{1}\left(\mathbf{x}_{\|}\right)}^{\psi_{2}\left(\mathbf{x}_{\|}\right)} d x_{3} \mathcal{L}_{I}(\varphi),
$$

where $x_{\|} \equiv\left(x_{0}, x_{1}, x_{2}\right)$ and $\mathcal{L}_{I}$ is a local Lagrangian.

In what follows, we assume that the $R$ medium is semitransparent, so that the corresponding term $\mathcal{S}_{R}$ can be treated perturbatively, to the first nontrivial order in $\lambda_{R}$. No assumption is made about the $L$ term.

The vacuum energy $U$ shall be a functional of the two functions $\psi_{1,2}$ and may be written in terms of the vacuum amplitude $\mathcal{Z}$, as

$$
U\left[\psi_{1}, \psi_{2}\right]=-\lim _{T \rightarrow \infty}\left(\frac{1}{T} \ln \mathcal{Z}\right),
$$

where $T$ is the extent of the imaginary time coordinate and

$$
\mathcal{Z}=\int \mathcal{D} \varphi e^{-\mathcal{S}(\varphi)} .
$$

Expanding the functional integral in powers of $\lambda_{R}$, the lowest nontrivial contribution reads

$$
U\left[\psi_{1}, \psi_{2}\right]=E\left[\psi_{1}\right]-E\left[\psi_{2}\right],
$$

with

$$
E\left[\psi_{1,2}\right]=\lim _{T \rightarrow \infty}\left[\frac{1}{T}\left\langle S_{1,2}[\varphi]\right\rangle_{L}\right] .
$$

Here we have introduced

$$
\mathcal{S}_{1,2}[\varphi]=\frac{\lambda_{R}}{2} \int d^{3} x_{\|} \int_{\psi_{1,2}\left(\mathbf{x}_{\|}\right)}^{\infty} d x_{3} \mathcal{L}_{I},
$$

and the $\langle\cdot\rangle_{L}$ symbol denotes the functional average with the weight defined by $\mathcal{S}_{0}+\mathcal{S}_{L}$. The crucial point is to observe that all the dependence with the shape of the surfaces is in the lower integration limit of $\mathcal{S}_{1,2}$. As a consequence, we can write

$$
U\left[\psi_{1}, \psi_{2}\right]=\int d^{2} \mathbf{x}_{\|}\left(E_{\|}\left(\psi_{1}\right)-E_{\|}\left(\psi_{2}\right)\right),
$$

where $E_{\|}(a)$ is the interaction energy per unit area between semispaces separated by a gap of width $a$. The physical interpretation of this result is that, as $E_{\|}$is the interaction between semispaces, in order to obtain the interaction energy for the configuration described by $\psi_{1}$ and $\psi_{2}$, one must subtract from $E_{\|}\left(\psi_{1}\right)$ the contribution coming from points with $x_{3}>\psi_{2}$. This "linearity" is valid only for the first order in $\lambda_{R}$. Equation (73) coincides with the result obtained using the SEI. 
In order to illustrate these points, let us assume that $\mathcal{L}_{I}=\varphi^{2}$. In the strong-coupling limit for $\lambda_{L}$, the field satisfies Dirichlet boundary conditions at $x_{3}=0$. An explicit calculation yields

$$
U\left[\psi_{1}, \psi_{2}\right]=\frac{\lambda_{R}}{32 \pi^{2}} \int d^{2} \mathbf{x}_{\|}\left[\frac{1}{\psi_{1}\left(\mathbf{x}_{\|}\right)}-\frac{1}{\psi_{2}\left(\mathbf{x}_{\|}\right)}\right],
$$

which is the difference between the PFA energies associated with the surfaces $\psi_{1}$ and $\psi_{2}$. It can be shown explicitly, however, that the property of the energy's being the difference between the ones corresponding to each surface is lost in the next order in $\lambda_{R}$.

To summarize this section, we have shown that SEI gives an exact result for almost-transparent media, where one can use the superposition principle and consider the interaction as the sum of pairwise potentials. Although this was mentioned in Ref. [6], the model presented here suggests how to go beyond the leading order and provides a systematic way of evaluating the interaction energy for dilute media. Finally, wenote that the fact that PFA becomes exact in this limit has also been mentioned by authors working in Casimir physics $[11,12]$.

\section{REVIEW OF RESULTS}

In this section we summarize and briefly review some of the results obtained through the use of the DE for the calculation of interaction energy between surfaces, in different contexts. It is our intention not to be exhaustive, but to enumerate some of the results that, we believe, are noteworthy. We also take the opportunity to pinpoint and clarify some aspects that deserve more comment than in their original presentation, under the light of this general derivation. For the Casimir effect, we consider a scalar field with Dirichlet and Neumann conditions, at zero and finite temperature, and also an electromagnetic field with non-perfectly-conducting surfaces. We show how the PFA emerges naturally from this approach and, also, calculate the NTLO correction to the PFA. We also review results obtained for the electrostatic interaction, for both surfaces at fixed potentials and surfaces endowed with patch potentials. At the end of this section we briefly discuss some findings related to nonanalytic terms appearing in the expansion at finite temperature in the Neumann case.

\section{A. Derivative expansion for the Casimir effect}

We have shown that the PFA can be thought of as being akin to the leading-order term in a derivative expansion of the Casimir energy with respect to the shape of the surfaces in Ref. [3]. Moreover, when the first nontrivial correction containing two derivatives of $\psi$ are included, the general formula gives the NTLO correction to the PFA for a general surface. The general expression for the second-order approximation to the interaction energy (or free energy, depending on the case) is the one shown in Eq. (17).

In Ref. [3] we applied the DE to the evaluation of the Casimir interaction energy for a scalar field with Dirichlet boundary conditions. The calculation consisted, in terms of the general derivations we present in Sec. II A of the present paper, of the application of the expansion to an effective action (proportional to the energy for static boundary conditions).
The DE was obtained by performing the same calculation as suggested in Sec. II A, namely, a second-order expansion of the functional around the parallel-plane case.

The general result for the DE approximation to the Casimir interaction energy for perfect mirrors can be written as

$$
U_{\mathrm{DE}}[\psi]=-\frac{\pi^{2}}{1440} \int d^{2} \mathbf{x}_{\|} \frac{1}{\psi^{3}}\left[\alpha+\beta(\nabla \psi)^{2}\right],
$$

where $\alpha$ and $\beta$ are numerical coefficients that depend on the field considered (scalar or electromagnetic) and on the boundary conditions imposed on the surfaces. This form for $U_{\mathrm{DE}}$ can of course be anticipated by simple dimensional analysis. The zeroth-order term equals the PFA to the vacuum energy, while the second-order term contains the first nontrivial correction to the PFA.

For a scalar field satisfying Dirichlet (D) boundary conditions [3] we have $\alpha_{D}=1$ and $\beta_{D}=2 / 3$. A scalar field with Neumann $(\mathrm{N})$ boundary conditions was considered in [13], where it was shown that $\alpha_{N}=1$ and $\beta_{N}=2 / 3\left(1-30 / \pi^{2}\right)$. In the same reference, the authors present the results corresponding to an electromagnetic field and perfectly conducting surfaces, which turns out to be the sum of the Dirichlet and Neumann results.

It is worth stressing the last result: within the DE approach, the electromagnetic Casimir interaction energy between perfectly conducting surfaces is the sum of the scalar Casimir energy for Dirichlet and Neumann boundary conditions. This is known for the leading PFA, and it is also valid for the first nontrivial correction [4]. Of course, it is not valid at higher orders.

Also in Ref. [13], the DE was extended to two curved surfaces, for Dirichlet, Neumann, mixed (Dirichlet and Neumann on different surfaces), and electromagnetic (perfect metal) boundary conditions. Reference [14] presents the leading correction to the PFA for gold at room temperature.

Although derived for surfaces describable by a single function $\psi\left(\mathbf{x}_{\|}\right)$, the DE has been applied to more general geometries that include compact objects in front of a plane. In these cases, the integration is restricted to a portion of the compact object that is closer to the plane. It has been shown that, for perfect mirrors, the PFA and its NTLO correction are insensitive to the choice of the integration area in the limit where the surfaces are very close [3]. This is not the case for semitransparent mirrors, as we have shown in the previous section.

In all particular examples where the NTLO correction to the PFA has been computed analytically, the results coincide with the prediction of the derivative expansion. This is the case for a cylinder in front of a plane [15] and also for a sphere in front of a plane [16]. Moreover, the DE has been useful to detect [14] an error in previous calculations [17] of the sphere-plane interaction energy beyond the PFA, which was subsequently corrected in Ref. [16].

Let us denote the exact Casimir interaction energy for a given geometry by $U$ and its PFA by $U_{\mathrm{PFA}}$. For both cylinderplane and sphere-plane geometries the analytic NTLO correction is of the form

$$
\frac{U}{U_{\mathrm{PFA}}}=1+\gamma \frac{a}{R},
$$

where $a$ is the minimum distance, $R$ the radius (of the sphere or the cylinder), and $\gamma$ a numerical coefficient that depends on the 
geometry and the boundary condition. For the cylinder-plane it has been shown that

$$
\gamma_{D}=\frac{7}{36}, \quad \gamma_{N}=\frac{7}{36}-\frac{40}{3 \pi^{2}},
$$

while for the sphere-plane

$$
\gamma_{D}=\frac{1}{3}, \quad \gamma_{N}=\frac{1}{3}-\frac{40}{\pi^{2}} .
$$

It has also been proved that the electromagnetic result is the sum of the Dirichlet and Neumann cases. All these results can be reproduced using the DE by plugging the functions $\psi$ corresponding to a cylinder and a sphere into Eq. (75) and expanding the result of the integrals in powers of $a / R$.

The numerical calculations are also consistent with the NTLO correction for the cylinder-plane geometry [18] and for the sphere-plane geometry [14], although for Neumann boundary conditions there is a discrepancy between the analytic predictions $[14,16]$ and the numerical fit. A similar discrepancy occurs with the fit presented in Ref. [19] for the electromagnetic case. We believe that these discrepancies may be due to the fact that the numerical calculations have not been performed for sufficiently small values of $a / R$, and therefore the fits are sensitive to the particular functions and intervals used to obtain them. This sensitivity has been noted in [18] for the cylinder-plane geometry and in [14] for the sphere-plane geometry.

\section{B. Patch potentials and the electrostatic energy interaction}

In Ref. [20], we have applied the DE to the evaluation of the electrostatic interaction energy (the functional to expand in derivatives) between two perfectly conducting surfaces, one flat and the other slightly curved, held at a potential difference $V_{0}$. In this situation, the interaction energy reads

$$
U_{\mathrm{DE}} \simeq \frac{\epsilon_{0} V_{0}^{2}}{2} \int d^{2} \mathbf{x}_{\|} \frac{1}{\psi}\left[1+\frac{1}{3}(\nabla \psi)^{2}\right] .
$$

We have shown explicitly that, in particular cases where analytic exact results are available, the DE reproduces the exact ones up to NTLO (this is the case, for instance, for a sphere or a cylinder in front of a plane).

In Ref. [21], we extended these results to the case in which the surfaces have patch potentials. These potentials were not introduced as boundary conditions but modeled by means of electric dipole layers that are adjacent to the surfaces. The result was expressed in terms of the two-point autocorrelation functions for those patch potentials and of the single function $\psi$ which defines the curved surface. The reason for studying this is based on the fact that surface imperfections can lead to a local departure from ideal metallic behavior, yielding a space-dependent patch potential on the surface of the mirrors. They produce a force that may be, in principle, relevant to the interpretation of precision experiments involving two surfaces.

In order to present a more compact expression for the results, it is convenient to assume that the potentials' autocorrelation function depends on the variance of the potential $V_{\text {rms }}$ and on a single characteristic length $\ell$. Then, on dimensional grounds we have that the Fourier transform of the auto correlation function is of the form

$$
\widetilde{\Omega}\left(\mathbf{k}_{\|}\right)=V_{\mathrm{rms}}^{2} \ell^{2} g\left(\left|\mathbf{k}_{\|}\right| \ell\right)
$$

for some dimensionless function of a dimensionless argument, $g$.

In terms of the objects above, we have found that

$$
V(\psi)=\frac{V_{\mathrm{rms}}^{2}}{\psi} v(\ell / \psi), \quad Z(\psi)=\frac{V_{\mathrm{rms}}^{2}}{\psi} z(\ell / \psi),
$$

where

$$
\begin{aligned}
v(\ell / \psi)= & -\frac{2}{\pi} \frac{\ell^{2}}{\psi^{2}} \int_{0}^{\infty} d x \frac{x^{2}}{e^{2 x}-1} g(x \ell / \psi), \\
z(\ell / \psi)= & \frac{1}{16 \pi} \frac{\ell^{2}}{\psi^{2}} \int_{0}^{\infty} d x \frac{x^{2} g(x \ell / \psi)}{\sinh ^{5}(x)}\left[\left(1-8 x^{2}\right) \cosh (x)\right. \\
& -\cosh (3 x)+12 x \sinh (x)] .
\end{aligned}
$$

One can show that, when $\ell \gg \psi, v$ and $z$ tend to the result for constant potentials, and therefore

$$
U_{\mathrm{DE}} \simeq-\epsilon_{0} V_{\mathrm{rms}}^{2} \int d^{2} \mathbf{x}_{\|} \frac{1}{\psi}\left[1+\frac{1}{3}(\nabla \psi)^{2}\right] .
$$

This is twice the result for the electrostatic energy between surfaces held at a constant potential difference $V_{\text {rms }}$ [see Eq. (79)]. The factor 2 comes from the fact that we are considering the same correlation function on both surfaces. ${ }^{2}$

On the other hand, in the opposite limit, $\ell \ll \psi$, one can make the approximation $g(x \psi / \ell) \simeq g(0)$ inside the integrals to get

$$
V \simeq-\frac{g(0) \zeta(3) \ell^{2}}{2 \pi} \frac{V_{\mathrm{rms}}^{2}}{\psi^{3}}, \quad Z \simeq-\frac{g(0)(1+6 \zeta(3)) \ell^{2}}{24 \pi} \frac{V_{\mathrm{rms}}^{2}}{\psi^{3}},
$$

which has the same dependence on distance as the Casimir energy, something which is, in this case, due to the lack of a dimensionful quantity associated with the correlation length.

\section{Derivative expansion at finite temperature}

In [22], we obtained expressions for the coefficients that determine the $\mathrm{DE}$ at finite temperatures, for the free energy $F$ in a Casimir system. ${ }^{3}$ We presented closed analytic expressions for these coefficients, in different numbers of spatial dimensions $d$, for both the zero- and the high-temperature limits. We considered surfaces satisfying either Dirichlet or Neumann boundary conditions, finding some qualitative differences between these two cases: for two Dirichlet surfaces, the NTLO term in the DE is well defined (local) for any temperature $T$. Besides, it interpolates smoothly between the proper limits: namely, when $T \rightarrow 0$ it tends to the one we had calculated for the Casimir energy, while for $T \rightarrow \infty$ it corresponds to

\footnotetext{
${ }^{2}$ There is also an extra minus sign, which comes from the fact that in the present calculation the potentials on the surfaces are not produced by external batteries but are due to the internal structure of the materials.

${ }^{3}$ We use here identical notation $(F)$ for the free energy and for the functional used in the general derivation of the DE.
} 
the one for a $d=2$ theory, realizing the expected dimensional reduction at high temperatures.

The DE approach (up to second order) may be applied to this case, with the free energy as a functional of the surface. We present the Dirichlet and Neumann cases separately.

\section{Dirichlet boundary conditions}

In the Dirichlet case, we write the Casimir free energy as

$$
\begin{aligned}
F_{\mathrm{DE}}[\psi]= & \int d^{d-1} \mathbf{x}_{\|}\left\{b_{0}\left(\frac{\psi}{\beta}, d\right) \frac{1}{\left[\psi\left(\mathbf{x}_{\|}\right)\right]^{d}}\right. \\
& \left.+b_{2}\left(\frac{\psi}{\beta}, d\right) \frac{(\nabla \psi)^{2}}{\left[\psi\left(\mathbf{x}_{\|}\right)\right]^{d}}\right\},
\end{aligned}
$$

where the two dimensionless functions $b_{0}$ and $b_{2}$ can be obtained from the knowledge of the Casimir free energy for small departures around the $\psi\left(\mathbf{x}_{\|}\right)=a=$ constant case. In the very-high (infinite)-temperature limit, we have that

$$
\begin{aligned}
& {\left[b_{0}(\xi, d)\right]_{\xi \gg 1} \simeq \xi\left[b_{0}(\xi, d-1)\right]_{\xi \rightarrow 0} \equiv \xi b_{0}(d-1), } \\
& {\left[b_{2}(\xi, d)\right]_{\xi \gg 1} \simeq \xi\left[b_{2}(\xi, d-1)\right]_{\xi \rightarrow 0} \equiv \xi b_{2}(d-1), }
\end{aligned}
$$

where $\xi=\psi / \beta$. The coefficients $b_{0}(d-1)$ and $b_{2}(d-1)$ are those corresponding to perfect mirrors at zero temperature in $d-1$ dimensions, a reflection of the well-known "dimensional reduction" phenomenon at high temperatures, for bosonic degrees of freedom. In particular, the DE up to the second order in the high-temperature limit, in $d=3$ dimensions, is

$$
\begin{aligned}
\left.F_{\mathrm{DE}}[\psi]\right|_{\psi / \beta \gg 1, d=3} \sim & -\frac{\zeta(3)}{16 \pi \beta} \int d^{2} \mathbf{x}_{\|} \frac{1}{\left[\psi\left(\mathbf{x}_{\|}\right)\right]^{2}} \\
& \times\left\{1+\frac{(1+6 \zeta(3))}{12 \zeta(3)}(\nabla \psi)^{2}\right\} .
\end{aligned}
$$

Let us apply this result to the evaluation of the Dirichlet Casimir interaction for a sphere in front of a plane. As before, we denote by $a$ the minimum distance between the surfaces, and by $R$ the radius of the sphere. As already mentioned, although the surface of the sphere cannot be covered by a single function $z=\psi\left(\mathbf{x}_{\|}\right)$, we consider just the region of the sphere which is closer to the plane [3].

The sphere is described by the function

$$
\psi=a+R\left(1-\sqrt{1-\frac{\rho^{2}}{R^{2}}}\right),
$$

where we have used polar coordinates $(\rho, \phi)$ for the $x_{3}=0$ plane. This function describes the hemisphere when $0 \leqslant \rho \leqslant$ $R$. The DE will be well defined if we restrict the integrations to the region $0 \leqslant \rho \leqslant \rho_{M}<R$.

We assume that $\rho_{M} / R=O(1)<1$. Inserting this expression for $\psi$ into the free energy, Eq. (87), and performing explicitly the integrations, we obtain ${ }^{4}$

$$
\left.F_{\mathrm{DE}}[\psi]\right|_{\psi / \beta \gg 1, d=3} \sim-\frac{\zeta(3) R}{8 \beta a}\left(1-\frac{1}{6 \zeta(3)} \frac{a}{R} \ln \left(\frac{a}{R}\right)\right) .
$$

\footnotetext{
${ }^{4}$ There is a typo in Eq. (39) in Ref. [22]. The coefficient 0.569 should read $-1 / 6 \zeta[3]$.
}

Note that, as long as $a \ll R$, the force will not depend on $\rho_{M}$. As expected on dimensional grounds, the $R / a^{2}$ behavior of the leading contribution in the zero-temperature case changes to $R / a \beta$ at high temperatures. This problem has been solved exactly in Ref. [23]. One can readily show that Eq. (89) coincides with the small distance expansion of the exact result.

It is interesting to note that the NTLO correction from the DE becomes nonanalytic, because of the integration, in the parameters defining the function $\psi$. This behavior has been noted in numerical estimations of the Casimir interaction between a sphere and a plane in the infinite-temperature limit, for the electromagnetic case in Refs. [23] and [24]. Note that this nonanalyticity has nothing to do with the nonanalyticity of the form factors described in Sec. II A (see also Sec. IV C below). There, the DE was not applicable; here we deal with terms that appear in a system where the DE is perfectly well defined. One integrates over the surface, and when expanding or with a small $a / R$, one gets both analytic and nonanalytic contributions. The latter are not a drawback but a normal feature of the DE.

Very recently, the free interaction energy between a sphere and a plate at high temperatures has been computed exactly in an arbitrary number of dimensions for Dirichlet boundary conditions [25]. We have checked that the DE reproduces the leading and NTLO of the exact result for $d=4$ and 5. We sketch the calculations here. In the high-temperature limit the free energy reads

$$
\begin{aligned}
F_{\mathrm{DE}}[\psi]= & \frac{1}{\beta} \int d^{d-1} \mathbf{x}_{\|}\left\{b_{0}(d-1) \frac{1}{\left[\psi\left(\mathbf{x}_{\|}\right)\right]^{d-1}}\right. \\
& \left.+b_{2}(d-1) \frac{(\nabla \psi)^{2}}{\left[\psi\left(\mathbf{x}_{\|}\right)\right]^{d-1}}\right\} .
\end{aligned}
$$

Inserting Eq. (88) into Eq. (90) and expanding in powers of $a / R$, we obtain, for $d=4$,

$$
\frac{F_{\mathrm{DE}}}{F_{\mathrm{PFA}}}=1+\frac{1}{4} \frac{a}{R},
$$

while for $d=5$ we get

$$
\frac{F_{\mathrm{DE}}}{F_{\mathrm{PFA}}}=1+\frac{1}{3}\left(1-\frac{\zeta(3)}{\zeta(5)}\right) \frac{a}{R} .
$$

Both expressions are consistent with the analytic results for the force presented in Ref. [25].

\section{Neumann boundary conditions}

The free energy can be written as before [see Eq. (85)], but with coefficients $c_{0}$ and $c_{2}$ instead of $b_{0}$ and $b_{2}$. The zero-order term coincides with the one for the Dirichlet case, namely, $c_{0}=b_{0}$. The second-order coefficient is given by

$$
c_{2}(\xi)=\frac{1}{2}\left[\frac{\partial G^{(2)}\left(\xi ; n,\left|\mathbf{I}_{\|}\right|\right)}{\partial\left|\mathbf{I}_{\|}\right|^{2}}\right]_{n \rightarrow 0,\left|\mathbf{I}_{\|}\right| \rightarrow 0} .
$$

The expression of $G^{(2)}\left(\xi ; n,\left|\mathbf{I}_{\|}\right|\right)$was calculated in [22]. We do not present the explicit expression of it here since their form is not relevant for the actual presentation.

For $d=1$, the coefficient $c_{2}$ coincides with its Dirichlet counterpart $b_{2}$. In higher dimensions, the structure of the NTLO correction is different. 
For $d=3$, the NTLO term contains, besides a standardlooking local term, also a nonlocal contribution, linear in $T$ and, therefore, present for any $T>0$. This leaves room, when the temperature is sufficiently low, to use just the local term (of second order in derivatives) as the main correction to the PFA.

Of course, the nonlocal term will always break down for a sufficiently high temperature, whose value will depend on the actual shape of the surface involved. We stress once more that this nonanalytic behavior is a consequence of the Neumann boundary conditions and may not be present for imperfect boundary conditions, such as those considered in Ref. [13]. This point deserves further analysis.

It is important to note that a local DE breaks down for Neumann boundary conditions at $d=2$ and zero temperature. However, one can still perform an expansion for smooth surfaces, including nonlocal contributions in the Casimir energy. For instance, in this case, the NTLO correction to the PFA will be nonlocal and proportional to

$$
\int d^{2} x_{\|} \eta\left(x_{\|}\right) \nabla_{\|}^{2} \ln \left(-a^{2} \nabla_{\|}^{2}\right) \eta\left(x_{\|}\right)
$$

where $\eta$ is a small departure from $\psi=$ const. The breakdown of the local expansions is related to the existence of massless modes in the theory. These modes are generally allowed by Neumann but not by Dirichlet boundary conditions, which impose a mass gap of order $1 / a$.

The logarithmic behavior of the form factor in $d=2$ induces a similar nonanalyticity for $d=3$ at finite temperature. Therefore, in an expansion for small values of $\left|k_{\|}\right|$, in addition to a term proportional to $k_{\|}^{2}$, there is a contribution proportional to $(T a) k_{\|}^{2} \ln \left(k_{\|}^{2} a^{2}\right)$ at any nonvanishing temperature, which is not canceled by the rest of the sum over Matsubara frequencies.

\section{CONCLUSIONS}

We have presented both a construction of the DE, based on a physical argument, and a formal derivation of it, for a general family of problems, which can be defined in terms of a functional depending on a function characterizing a surface as its argument. This can be applied, as it has been, to Casimir and electrostatic problems. We have argued that the same procedure can also be used in nuclear and colloidal physics, since the derivation is sufficiently general to encompass these and other physical situations, such as Casimir-like forces in critical systems [26]. We have made contact with the latter by comparing and putting in similar terms the various existing
PFA-like approximations, showing that they correspond to the zeroth order in the DE.

The existing results about the application of the DE to different contexts have been briefly reviewed, mentioning some of the features that, we believe, may shed new light on the respective systems. We have shown in an explicit example, how the DE may induce nonanalytic contributions to the ratio $a / R$, where $a$ is the minimum distance and $R$ the radius of a sphere, for the Casimir free energy between a plane and a sphere at high temperatures. This nonanalyticity is dependent on the geometry of the system considered and appears in situations where the DE is well defined. In other words, it is not due to the existence of nonanalyticities in the momentum kernel of the second functional derivative of the functional $F$.

We have shown that the DE does reproduce correctly the NTLO corrections in various Casimir calculations. This is the case, in particular, for the sphere-plane geometry with Dirichlet boundary conditions at very high temperatures (the classical limit), where the result is known exactly in an arbitrary number of dimensions.

We end this paper with a few remarks on the generality of the DE approach and possible future lines of research. Regarding the interactions, the DE can be applied, in principle, both to additive and to nonadditive forces, superficial or volumetric, as long as the interaction energy can be written as a functional of the geometry of the surfaces. This is the case when the surfaces describe homogeneous physical objects with very small widths or when they correspond to interfaces between different homogeneous material media. There are of course situations where the above condition is not met. For instance, the gravitational interaction between two nonhomogeneous bodies cannot be described as a functional of their shapes.

Regarding the geometry of the bodies, up to now all applications of the DE have been restricted to a particular class of geometries, i.e., surfaces describable by functions $x_{3}=\psi\left(x_{1}, x_{2}\right)$, where $x_{i}$ are Cartesian coordinates. It would be interesting to generalize the results to other coordinates or, even better, to provide a covariant formulation in terms of geometric invariants of the surfaces. Work in this direction is in progress.

\section{ACKNOWLEDGMENTS}

F.D.M. would like to thank D. Dalvit, T. Emig, F. Intravaia, A. Lambrecht, P. Maia Neto, and S. Reynaud for discussions on this and related matters during the workshop Casimir Physics 2014. We would also like to thank D. Dantchev for useful comments regarding the SEI and SIA. This work was supported by ANPCyT, CONICET, UBA, and UNCuyo.
[1] B. V. Derjaguin, Koll. Z. 69, 155 (1934); B. V. Derjaguin and I. I. Abrikosova, Sov. Phys. JETP 3, 819 (1957); B. V. Derjaguin, Sci. Am. 203, 47 (1960).

[2] J. N. Israelachvili, Intermolecular and Surface Forces (Academic Press, London, 1992); P. W. Milonni, The Quantum Vacuum (Academic Press, San Diego, CA, 1994); M. Bordag, G. L. Klimchitskaya, U. Mohideen, and V. M. Mostepanenko, Advances in the Casimir Effect (Oxford University Press, Oxford, 2009).
[3] C. D. Fosco, F. C. Lombardo, and F. D. Mazzitelli, Phys. Rev. D 84, 105031 (2011).

[4] C. D. Fosco, F. C. Lombardo, and F. D. Mazzitelli, Phys. Rev. D 85, 125037 (2012).

[5] S. Bhattacharjee and M. Elimelech, J. Colloid Interface Sci. 193, 273 (1997).

[6] D. Dantchev and G. Valchev, J. Colloid Interface Sci. 372, 148 (2012). 
[7] J. Blocki, J. Randrup, W. J. Swiatecki, and C. F. Tsang, Ann. Phys. (NY) 105, 427 (1977).

[8] J. Blocki and W. J. Swiatecki, Ann. Phys. (NY) 132, 53 (1981).

[9] For an explicit calculation up to the fourth order in the context of Casimir physics, see C. D. Fosco, F. C. Lombardo, and F. D. Mazzitelli, Phys. Rev. D 86, 125018 (2012).

[10] I. Dutt and R. K. Puri, Phys. Rev. C 81, 064609 (2010).

[11] R. S. Decca, E. Fischbach, G. L. Klimchitskaya, D. E. Krause, D. Lopez, and V. M. Mostepanenko, Phys. Rev. D 79, 124021 (2009).

[12] K. A. Milton, P. Parashar, J. Wagner, and K. V. Shajesh, in Doing Physics: A Festschrift for Tom Erber, edited by P. Johnson (IIT Press, Chicago, 2011), pp. 211-224.

[13] G. Bimonte, T. Emig, R. Jaffe, and M. Kardar, Europhys. Lett. 97, 50001 (2012).

[14] G. Bimonte, T. Emig, and M. Kardar, Appl. Phys. Lett. 100, 074110 (2012).

[15] M. Bordag, Phys. Rev. D 73, 125018 (2006).

[16] M. Bordag and V. Nikolaev, Phys. Rev. D 81, 065011 (2010).
[17] L. P. Teo, M. Bordag, and V. Nikolaev, Phys. Rev. D 84, 125037 (2011).

[18] F. C. Lombardo, F. D. Mazzitelli, and P. I. Villar, Phys. Rev. D 78, 085009 (2008).

[19] A. Canaguier-Durand, P. A. Maia Neto, I. Cavero-Pelaez, A. Lambrecht, and S. Reynaud, Phys. Rev. Lett. 102, 230404 (2009).

[20] C. D. Fosco, F. C. Lombardo, and F. D. Mazzitelli, Ann. Phys. 327, 2050 (2012).

[21] C. D. Fosco, F. C. Lombardo, and F. D. Mazzitelli, Phys. Rev. A 88, 062501 (2013)

[22] C. D. Fosco, F. C. Lombardo, and F. D. Mazzitelli, Phys. Rev. D 86, 045021 (2012).

[23] G. Bimonte and T. Emig, Phys. Rev. Lett. 109, 160403 (2012).

[24] A. Canaguier-Durand, G. L. Ingold, M. T. Jaekel, A. Lambrecht, P. A. Maia Neto, and S. Reynaud, Phys. Rev. A 85, 052501 (2012).

[25] L. P. Teo, arXiv:1404.1419 [hep-th].

[26] See M. Labbé-Laurent, M. Tröndle, L. Harnau, and S. Dietrich, Soft Matter 10, 2270 (2014), and references therein. 